\title{
Wireless Sensors-Based Network to Measure Different Power Quality Disturbances
}

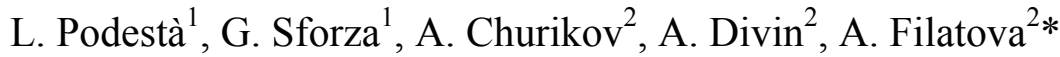 \\ ${ }^{1}$ University "La Sapienza”, 5, Aldo Moro, Piazzale, Roma, 00185, Italy \\ ${ }^{2}$ Department of Mechatronics and Technological Measurements, Tambov State Technical University, \\ 5, Kommunalnaya St., Tambov, 392000, Russia \\ * Corresponding author: Tel.: + 7 (4752) 6308 70.E-mail: Filang90@mail.ru
}

\begin{abstract}
Studies in the field of Power Quality have become increasingly important in recent years. International standards establish the limits of the main disturbances that can compromise the quality of electricity. According to GOST 12109-97, the voltage value at the final consumer should be $220 \mathrm{~V}$. Allowable voltage deviations are within $\pm 5 \%$, i.e. within $209 \mathrm{~V}$. The maximum permissible short-term voltage deviations can be $\pm 10 \%$, i.e. in the range of $198-242$ V. All voltage values outside these tolerances do not correspond to GOST 12109-97 and can be hazardous to appliances and equipment in one form or another. The project is related to the development of a sensors' network to measure Power Quality (with the ability to connect it both wirelessly and by wiring), for the analysis of a low-voltage grid. A wireless smart sensor which includes a transducer converting the value of the physical quantity to be measured into an electrical signal, a circuit for signal amplifying and conditioning, analogue/digital converter and a microcontroller has been developed. The RDF node for measuring basic parameters of power quality which allows connecting to the central computer which can detect the direction of sag events has been developed.
\end{abstract}

\section{Keywords}

Power Quality; smart sensors; low-voltage grid; EN 50160 standard; voltage sags measurement; disturbances of power quality.

(C) L. Podestà, G. Sforza, A. Churikov, A. Divin, A. Filatova, 2017

\section{Introduction to the Problems of Power Quality}

Electricity is the main "raw material" for industry and service sectors, and it is an example of the philosophy of "Just in time", according to which the components are delivered from a trusted supplier to the production line only when it is used, without making any preventive control, which ensures their quality before use. In fact, electricity is very different from any other product: it is usually generated away from the point of use, placed in a network fed by many other generators and arrives to the point of delivery through many different transformers and many kilometers of overhead lines and underground cables. To ensure the quality of the energy supplied to the point of delivery is not easy, and there is no way to withdraw electricity from the supply chain, which does not adequately meet the minimum requirements, or which is refused by the customer.

From the user point of view the problem is even more complex, since it can reveal inefficiencies, such as the complete failure, which can last from several seconds to several hours, or sags, during which the voltage drops for a short time at a level lower than nominal. A cause of troubles is not only long interruptions. Some processes are profoundly affected even by very short breaks, for example:

- continuous processes, during which brief interruptions can alter the synchronization of the machines and lead to large amounts of products not fully worked;

- operations linked to multiple levels of processing, where an interruption during a process can affect the result of other operations; 
- data Processing, in which the value of the transaction is high, while the cost of the process is low. This happens for example in managing actions, and currency exchange.

Each of the problems related to the quality of energy is caused by distinct phenomena. Some problems come from sharing the power supply network by multiple users, others are born in facilities of the users themselves and may or may not propagate through the network with consequences against other customers. The concept of quality of energy is not easy to define there is not a single parameter, which can describe the complete and immediate change in it.

The UNI ISO 8402 specifications dictate the quality of a generic product: "Quality is the set of properties and characteristics of a product or service that give it the ability to meet the expressed or implied needs" [1]. The problem arises when it is necessary to apply such a general definition to a particular product such as electricity.

It is possible however to define the "energy quality" as a perfect supply of it, which can ensure continuity of service, within certain tolerances of voltage and frequency, and can be characterized by a voltage with undistorted sinusoidal waveform. The acceptability of the deviations from the nominal characteristics of the energy depends on the type of use by the user, the equipment installed and its needs.

The quality of electricity that a typical user considers necessary for his activity is not therefore an absolute concept, but will depend on the users' sensitivity to noise (technical aspect) and the consequences of disruption (economic aspect) being thus variable from case to case. In these terms then the quality of the energy falls within the terms outlined by the general rule cited above, with the difference that the responsibility for the fulfillment of the quality requirements depends only partially on the manufacturer/distributor and the user/consumer is able to modify the qualitative characteristics of the instant purchasing power and then uses the product electricity.

In conclusion, the disturbances that affect the user depend on the characteristics of the distribution network, and on the behavior and the sensitivity of their loads. In Table 1, there are reported both disturbances aforesaid, with their tolerances compared to standard EN $50160[2,3]$.
Voltage distortion depends on the distance from the transformer and on the line impedance. In short, the voltage distortion affecting the grid at a certain location depends on the value of the short-circuit current of that point. Also, once the grid voltage is distorted, a linear load absorbs a distorted current. The presence of such harmonics on the grid is responsible for detrimental effects. Moreover, at higher frequencies, iron losses (hysteresis losses and eddy current losses) as well as the losses in the cables increase. Finally, electronic equipment may experience failures due to the presence of harmonics.

Another aspect, which should not be neglected, is the resonance issue related to the presence of harmonics in electrical networks. In fact, in this case the amplitude of a specific harmonic may increase several times as much of normal operation. Consequently this high-value current may seriously damage capacitors and equipment connected to the grid.

In order to prevent this kind of event, the resonance frequency of the grid at a certain point has to be known and, additionally, the insertion of wellfitted anti-resonance coils may be considered to damp the oscillatory phenomena.

The purpose of the project is to create a network that can monitor systematically the power quality of a site.

\section{Introduction to Smart Sensors}

Intelligent sensors are used in a growing number of industrial applications in which, with the development of innovative architectures, the transducer participates in the elaboration of the overall data, which in these new configurations can be divided between all the elements $[4,6]$. Fig. 1 shows the structure that characterizes these devices, based on the presence inside the transducer of the following functional blocks:

Table 1

Table with tolerance of disturbances on the network, according to standard EN 50160

\begin{tabular}{lc}
\hline \multicolumn{1}{c}{$f_{n}$ (interconnected systems) } & $50 \mathrm{~Hz} 1 \%(95 \%$ of time $)$ \\
\hline$V_{n}($ dal 2003$)$ & $50 \mathrm{~Hz}+4-6 \%(100 \%$ of time $)$ \\
Flicker & $230 / 40010 \%(95 \%$ of time $)$ \\
Voltage dips $(10 \mathrm{~ms}-1 \mathrm{~min})$ & $P l<1(95 \%$ of time $)$ \\
Short interruption $(<3 \mathrm{~min})$ & $<1000$ a year \\
Long interruption $(>3 \mathrm{~min})$ & Hundreds a year \\
Voltage harmonics & $<50$ a year \\
\hline
\end{tabular}




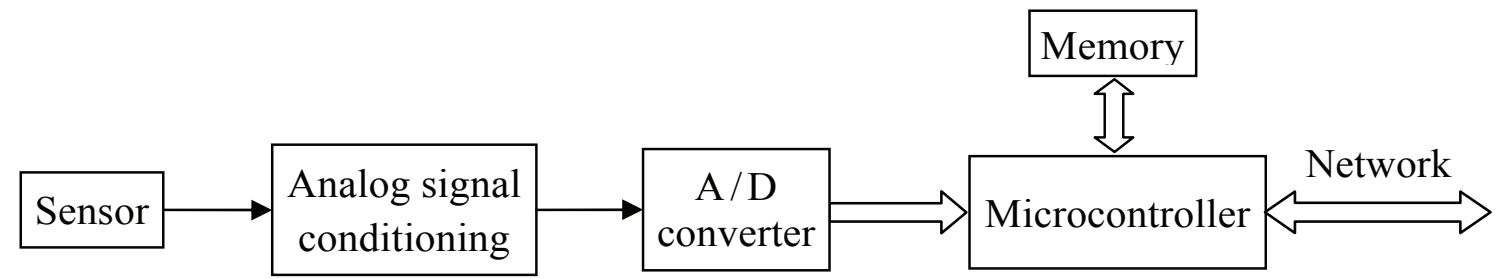

Fig. 1. Block diagram of an intelligent sensor

- sensor/transducer, which converts the value of the physical quantity to be measured into an electrical signal;

- circuit (usually analogue), for the amplification and signal conditioning;

- analog/digital converter that converts the signal into a digital format;

- microcontroller with its memory that contains both data which are detected, and the program for data management;

- communication interface to the outside.

The structure of a smart sensor includes, therefore, an element that should be able to convert the value of the physical quantity to be detected in information of an electrical nature, which is converted into numerical form in order to facilitate the management and processing by the microcontroller.

\section{Quantities to Be Measured}

At the point of measurement it is necessary that the instrument detects the following parameters: frequency, voltage, current and $\cos \varphi$, from which it will be possible to obtain the value of the various derived quantities including the reactive power. In this regard, data processing complies with the requirements of IEC 61000-4-30 [1]. For example, it complies with the requirement that the measurement uncertainty associated with the measurement frequency must not exceed $\pm 50 \mathrm{mHz}$ in the measurement fields $\sim 42.5 \mathrm{~Hz}$ $57.5 \mathrm{~Hz} / 51 \mathrm{~Hz} \sim 69 \mathrm{~Hz}$, the measurement uncertainty associated with the amplitude of the supply voltage should not be more than $\pm 0.5 \%$ of Udin, in the range $20 \% \sim 120 \%$ of Udin (Udin input voltage declared value obtained from the supply voltage declared by a ratio of the transducer). And so on for the other measured quantities, the goal was to create a device belonging to the class $\mathrm{S}$.

To identify the source of a voltage dip it is first necessary to evaluate the intensity of the event through its features and unambiguously define its duration, depth and instant start. This latter fact is important to investigate other parameters monitored and perform a matching. The starting instant of the event is the instant in which the rms value of the measured voltage becomes less than the threshold value of voltage used as a reference, in the case of monitoring a voltage dip, and not of a simple lowering. Such value is equal to $90 \%$ of the rated voltage. With regard to the classification requirements of the sag we followed the industry standards (IEC 50160, 1159). On the measurement point the operating characteristics of the node you are monitoring are acquired in real time: voltage, current, active and reactive power, THD, network frequency (the calculation of the frequency and THD are the result of the implementation of the software PLL or DPLL [7]). The calculation of the phase follows the same logic of the frequency detector of the PLL VCO where the samples were replaced by the current samples measured: in this way it is possible to take advantage of already written code for the calculation of the phase. Having the phase it is particularly easy to derive various values of active, reactive and apparent power.

Once the event has been identified, it is possible to analyze the variation of reactive power in transit. This change should be assessed by comparing the value of reactive power in the system, i.e. before the event, and that during the disturbance.

This method has been implemented within a network of sensors for a distributed measurement, and it will be illustrated in more detail in the following paragraphs.

\section{Prototype of the Node}

One of the objectives pursued in the design was to obtain the flexibility of the module, in order to be used for different measurements. It is possible, by changing a few lines of code, to implement functions that return a set of parameters than those currently produced.

The prototype realized at an early stage allowed us to perform the first steps and check the feasibility of some points. It is currently being developed on a DSPbased device that allows a performance improvement in terms of computational and considerable possibility of miniaturization also allowing the use of a single processor. If you need to store a greater amount of data, for non-real time query, you can add an external 


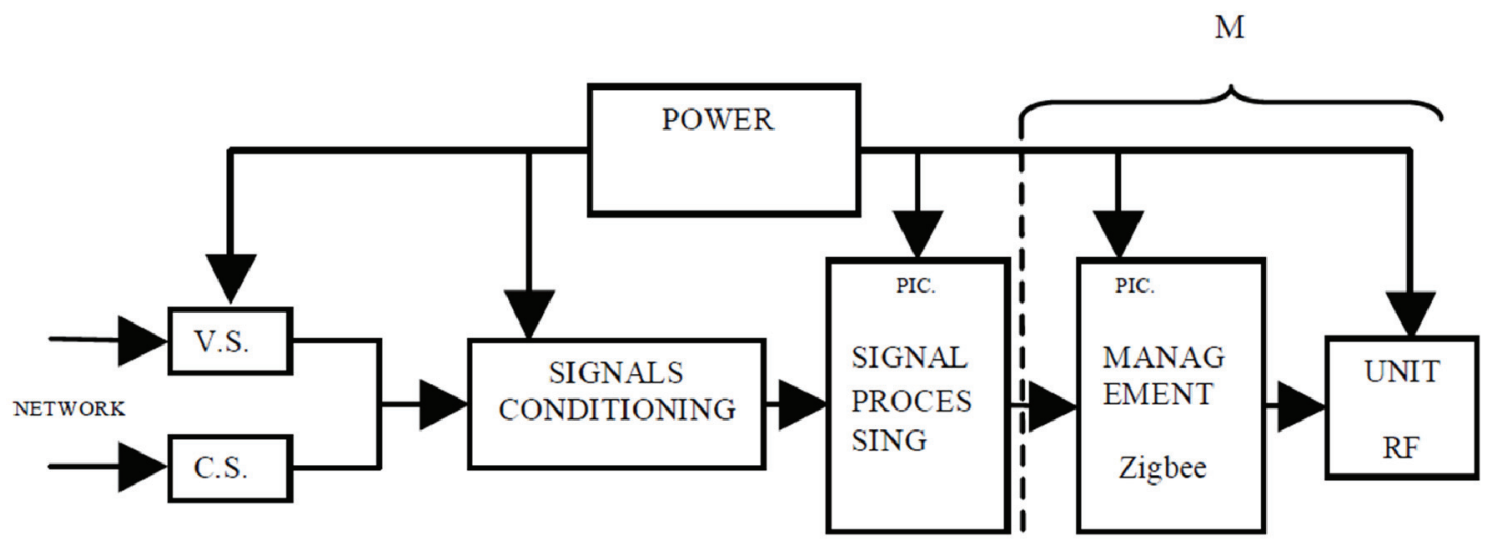

Fig. 2. Block diagram of the wireless sensor for power quality measurements

EEPROM to the device (this requirement has already been incorporated in the target of the second prototype in development).

The block diagram of the device is shown in Fig. 2.

The design of the power supply is surely one of the most delicate aspects to be taken into account when developing a measurement circuit. The voltage stability is critical both for all those devices which for their proper functioning requires a stable operating point, as the operational amplifiers that constitute the part of signal conditioning, or regarding to the sampling, as voltage variations influence the amplitude quantization, and then the entire measurement would be altered. In this case, in addition to stability, a high immunity to all line disturbances is required, disturbances which presumably will be the ones that the sensor must detect and analyze.
The specifications of the project in this case are:

- dual supply voltage $\pm 12 \mathrm{~V}$ necessary for the voltage sensor;

- supply voltage of $+5 \mathrm{~V}$ for the current sensor, for serial communication with the PC and for the processor computing;

- supply voltage of $+3.3 \mathrm{~V}$ for the processor that takes care of the management part of the Zigbee, and for the card to RF;

- total absorption of about $60 / 70 \mathrm{~mA}$ in transmission or in reception.

The circuit is shown in Fig. 3.

For the acquisition of voltage and current signals, we used two Hall effect sensors. Due to their characteristics, the input signal is faithfully reproduced, also ensuring excellent insulation from the network. The acquired signals are processed to be adapted to the input levels of the processor, as shown in the diagram (Fig. 4).

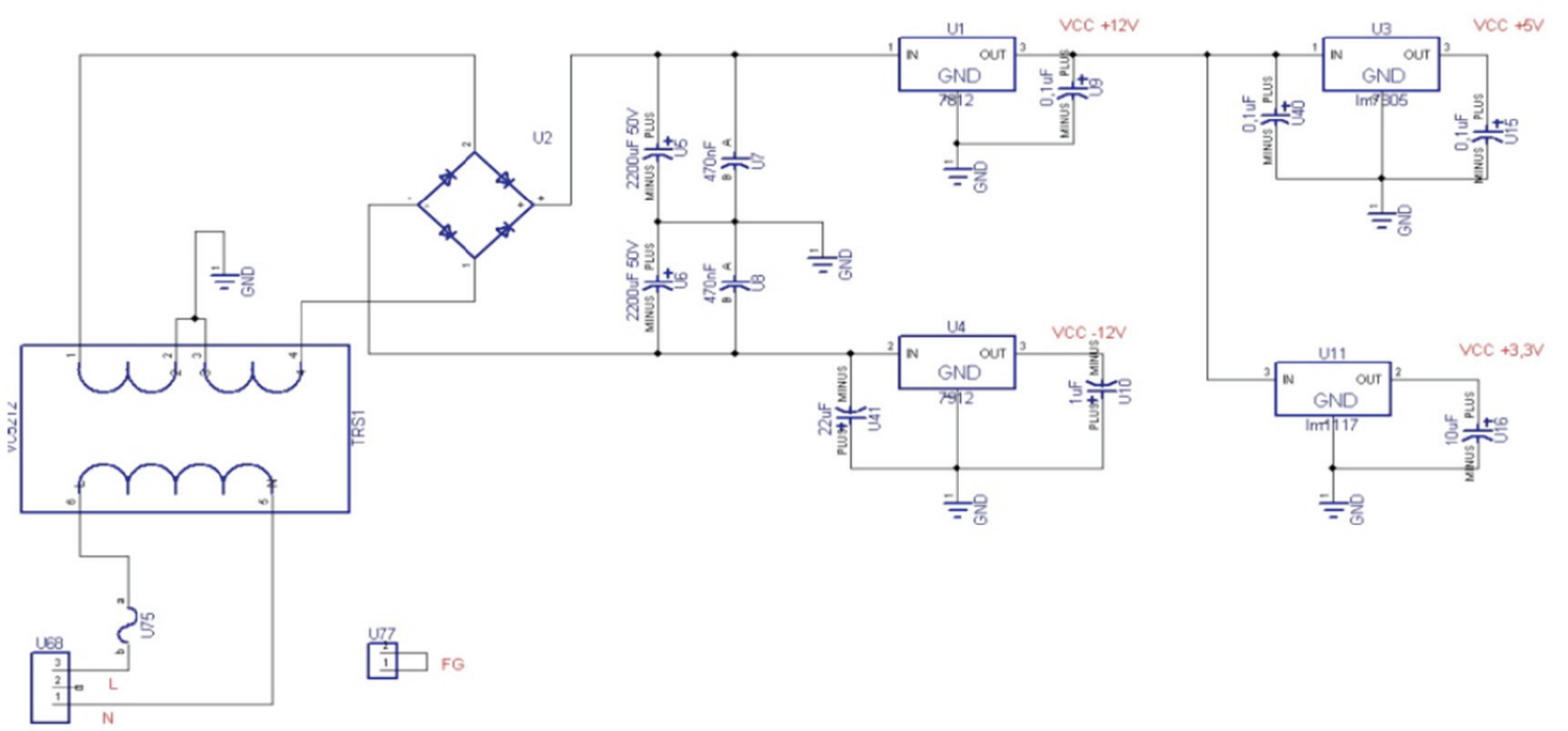

Fig. 3. Circuit diagram of the feeding part 


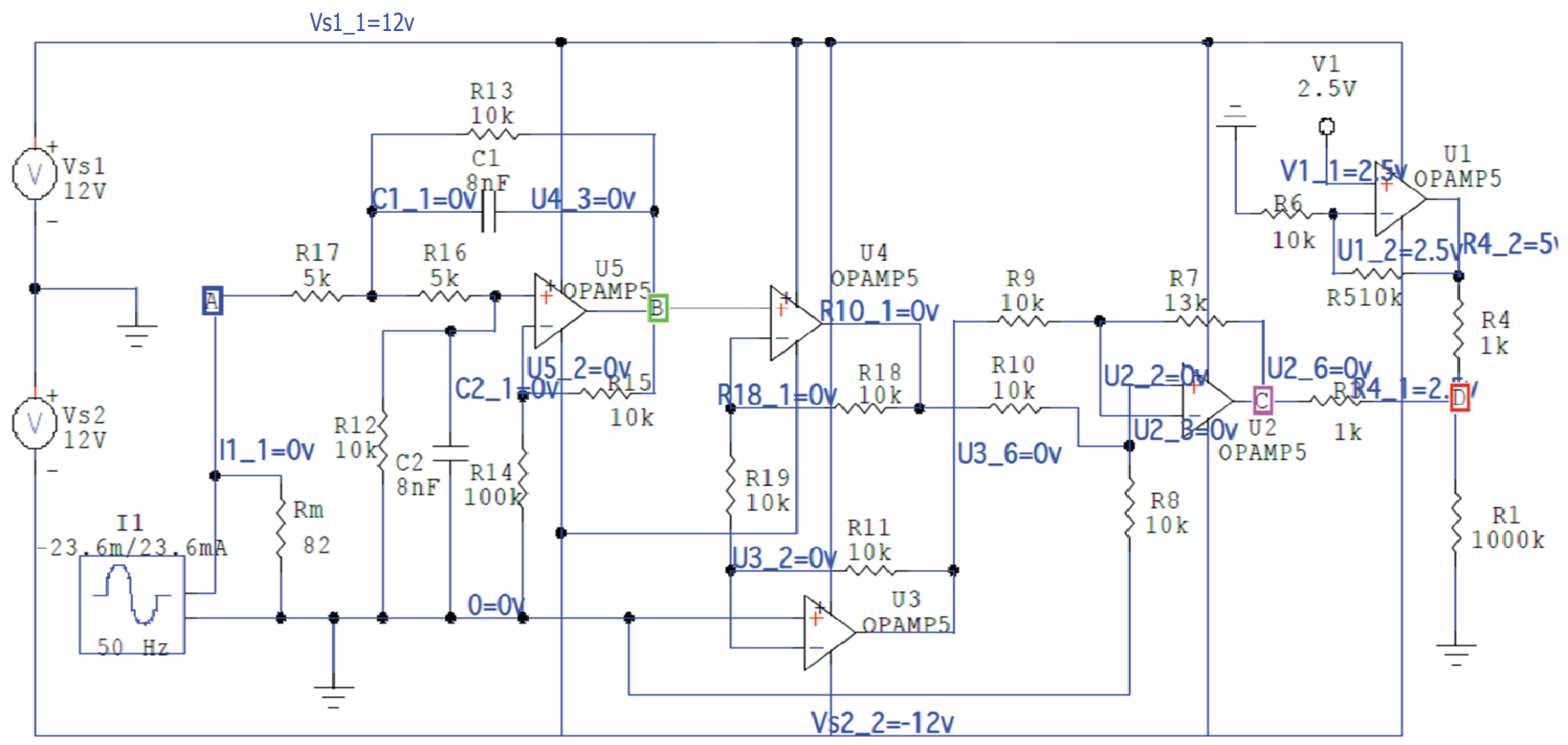

Fig. 4. Schematic diagram of the circuit for the treatment of the voltage signal (implemented with the program CircuitMaker)

The next stage of processing is handled by 18F4620 microcontroller. Here all the signal processing is performed, pre-treated and sampled at a frequency of $4 \mathrm{kHz}$, which is necessary for the study up to the 40th harmonic, as indicated by the CEI EN 50160. For communication between two microcontrollers a serial protocol has been implemented between pin $25(t x)$ of the processing pic, and pin $26(r x)$ of the network management pic. The protocol is very simple: the data to be transmitted are stored in a structure composed of 42 bytes. The first byte is a start byte, then, there are 40 bytes that represent the data. Finally a parity byte is sent. It has also added a switch between the two serial outputs of the pic so you can monitor via the PC the

Table 2

\section{Details of the absorption of the parts of the prototype}

\begin{tabular}{lc}
\hline \multicolumn{1}{c}{ Device } & Current consumption, $\mathrm{mA}$ \\
\hline Voltage sensor & $\sim 20$ \\
Current sensor & $\sim 30$ \\
PIC18F4620 & $\sim 2.5$ \\
Analog processing & $\sim 3$ \\
Card RF tx,rx & $\sim 18$ \\
Total & $\sim 74$ \\
\hline
\end{tabular}

outputs generated by both. The serial communication to the $\mathrm{PC}$ is made possible according to the standard that describes the RS232 specifications. The transmission stage consists of a dedicated pic (of the same family of the processing pic), which is implemented by the transmission protocol of ZigBee. Finally a CHIPCOM SmartRF CC2420 transceiver has been used, designed for applications with low energy consumption operating in the ISM band of $2.4 \mathrm{GHz}$.

In a Wireless Personal Area Network (WPAN) a Full Function Device (FFD) device can communicate with all devices in the network, while an Reduced Function Device (RFD) can only be associated with a FFD. In Fig. 6 the Sensors for Power Quality (SPQ) are RFD nodes, while the router is a FFD device.

In the study of consumption of the smart-sensor the three phases of measurement, the processing and the transmission of the data packet were analyzed separately. The following are the most interesting values (Table 2).

The sensors and the RF card parts are more expensive to manage. Even if this prototype is powered by the network you are trying to reduce consumption considerably by working on the sensors, in order to be independent from the network supply.

In Fig. 5 you can see the realization of the measurement point, the node of the network. The router is connected directly to a PC via cable, but in the next phase of the project a different connection, for example GSM, will be implemented to send warnings to the control stations. 


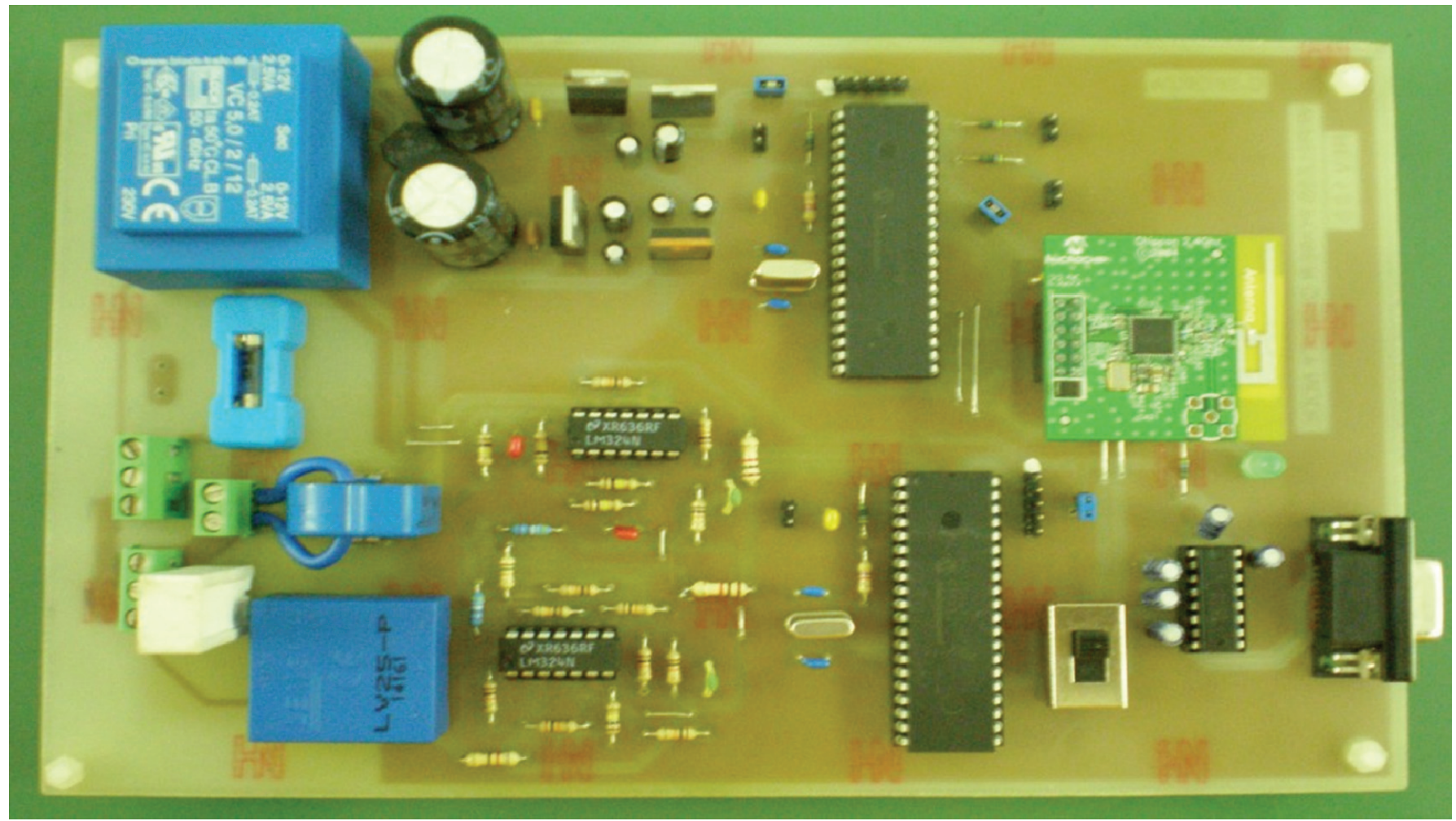

Fig. 5. Photo of the RFD node for power quality measurements

This single device realizes the SPQ block of the network shown in Fig. 6. The router is a device consisting only of much smaller processor that manages the ZigBee protocol and the RF card.

\section{Data Presentation}

The prototype has been used to perform some measurements within the university network. The tests were done in order to verify the parameters of robustness and reliability of the measuring instrument. Among the various tests that have been performed, the case of load insertion and disconnection on the network at the point of measurement is reported. As you can see the first node $796 \mathrm{~F}$ brings the values of the network at the point of measurement, and the variables related to the load from which it is possible to obtain a complete characterization: the current consumption, the phase shift introduced (almost resistive), the active, reactive and apparent power.

Fig. 7 shows a sample screen of what appears in real time on the PC that is connected to the Router. As you can see the network parameters are constantly measured and the required parameters are calculated.
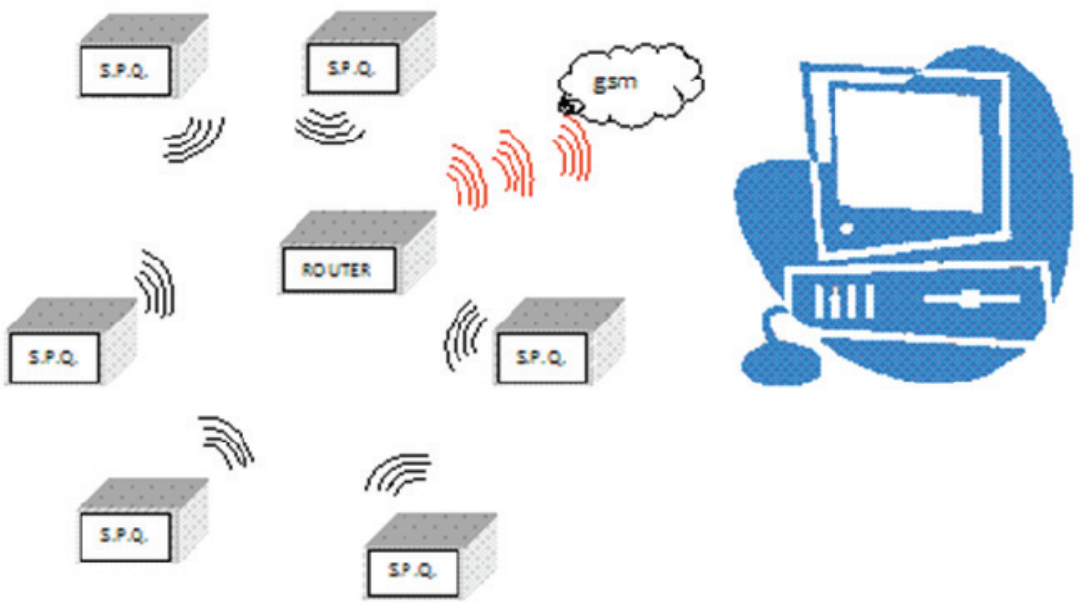

Fig. 6. Example of a wireless network for the measurement of power quality

\section{Algorithm for the Detection of Sags}

Among the most significant disturbances, due to the frequency with which they occur and the economic consequences that may result in, it is possible to recognize the phenomenon of voltage sags. The voltage sags are defined as a reduction of between 10 and $90 \%$ of the rms voltage at the frequency of operation for a period between 0.5 cycles and 1 minute. The sags may affect one or more stages depending on the phenomena that cause them. It is possible to ascribe the voltage drops to two main categories of 


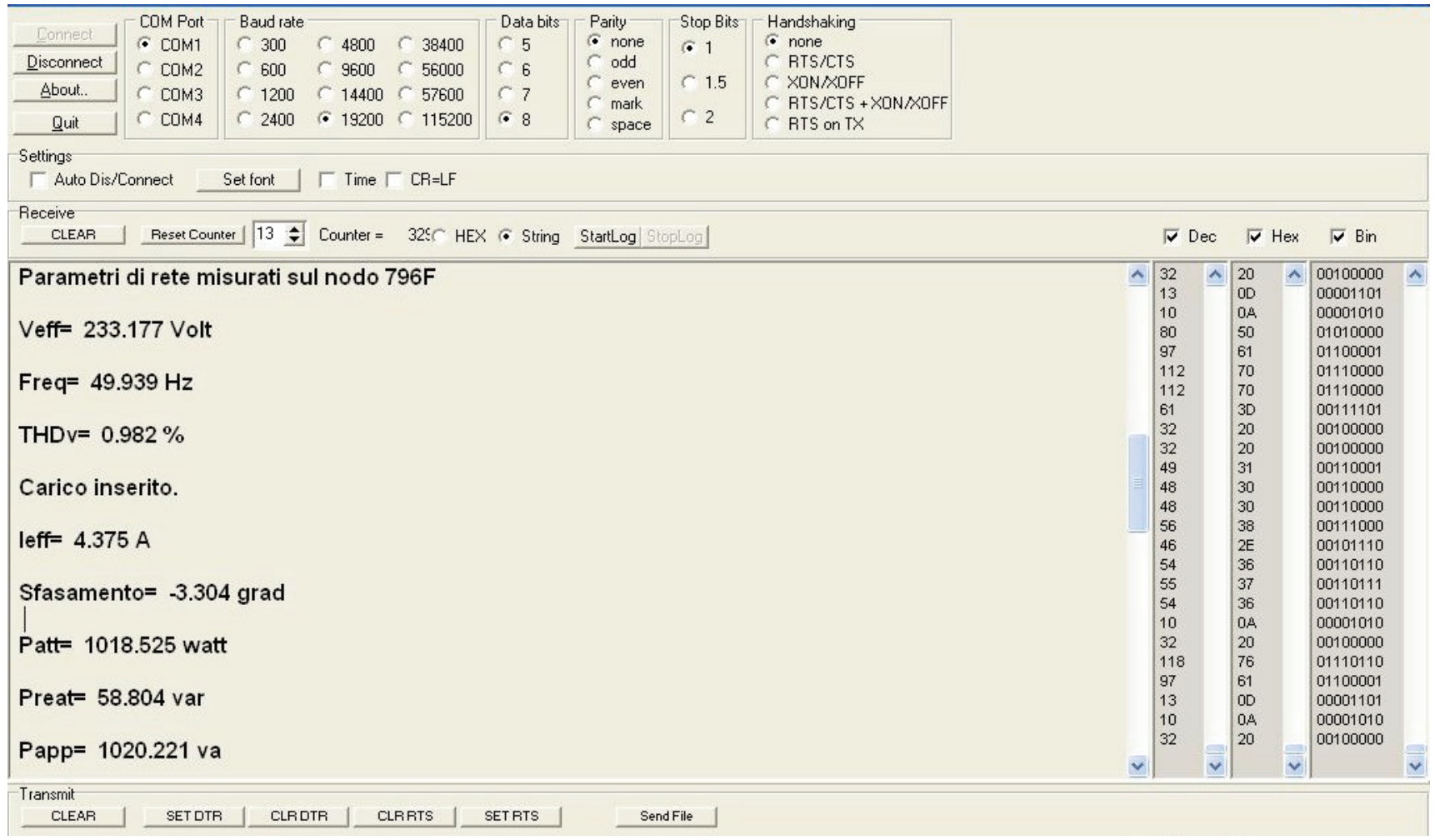

Fig. 7. Snapshot of a monitoring page of a measurement point

events: system failures, and overcurrents caused by maneuvering loads (loads insertion and start-up of motors, capacitor banks disconnection).

The main cause of voltage sags in a public network is represented by faults and in some cases by the transients overloads occurring at start up of large motors or during the insertion of large loads. These latter types of sag are usually due to the supplied loads while faults are associated with the interruption of the network.

In particular, the voltage sag is due to:

- transit in the network of a large current value in the case of a short circuit to ground or between the phases (remember the $\mathrm{ABC}$ classification, which distinguishes seven types of sags depending on the type of fault, on the transformer through which the current circulates and on the type of supplied load);

- demand of a high value of active and reactive power in the case of heavy loads insertion, such as an asynchronous motor or a heater based on Joule effect;

- demand of a high value of the inrush current in the case of startup of an engine and further increase of the reactive power absorbed due to the low initial value of the power factor of the motor (a power factor of 0.85 in the system can get to values of 0.45 or even 0.35 );

- demand of reactive power by a load with low power factor, in the case of sudden shutdown of the bank of capacitors used for power factor correction.

All the events mentioned above, at a certain instant, cause an increase in the current in the network.
For example, the electric motors typically absorb between 150 and $500 \%$ of the rated current until they reach their nominal speed, while heaters typically absorb $150 \%$ of the rated current until they arrive at the required temperature.

Because of the longitudinal impedance of the transmission line or distribution, the circulating current produces a voltage drop which can lead to voltage sags more or less important for the user. Also, a ruined or defective wiring increases the impedance of the system and therefore it amplifies the phenomenon.

Overcurrents contribute to the lowering of the voltage level because the latter is a function of the voltage drops through the components of the network. The effects of voltage sags are the malfunction of the devices connected to the network or the termination of the operation, depending on the extent of the event, in terms of depth and duration, and the limits of susceptibility of loads. It is therefore essential to locate the origin of the disturbance in order to allocate responsibilities, and possibly proceed with the correction of the causes.

Starting from the scientific literature and regulations it has been possible to define a simple and effective method for the localization of the sags in a low-voltage grid, with a commitment to produce an algorithm in a wireless sensor network with low cost and low impact [8]. The starting method is the study of the flow of reactive power [9]. 
The method of monitoring the reactive power flows tries to locate the origin of a voltage dip, indicating whether this is upstream or downstream relating to the point of observation. An event may be defined caused downstream, if set the direction of the active power flow, it is detected in the side to which the power flows, compared with the monitoring point, conversely, an event could be said to be originated upstream, if it is detected in the side from which the active power is supplied.

The reactive power, which is defined as $Q=V I \sin \varphi$, is monitored.

Since most of the passive components introduce an inductive phase shift, then $I \sin (\varphi)>0$, i.e. the reactive power in transit will be positive and concordant with the direction of current flow, and it is considered to be absorbed by the load. For a large part of the phenomena voltage drops correspond to an increase in the current in transit within the system. In these cases if the event takes place downstream of the measurement point, this will correspond to an increase in the current in transit and also to the increase in the product $I \sin \varphi$, and then also to an increase in the instantaneous power associated to the current component in quadrature with the voltage, whose peak value coincides with the

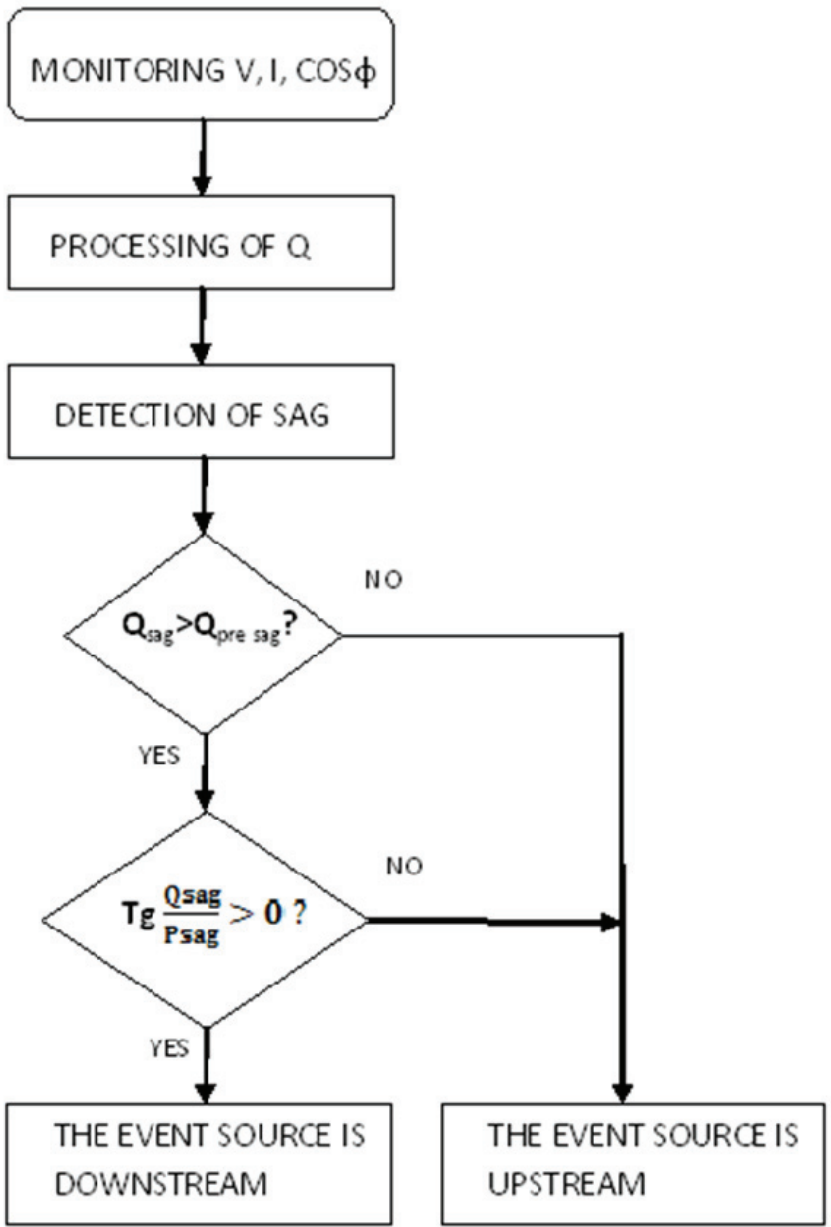

reactive power. The absorbed reactive power, taking the active power flow as a reference, will undergo an increase. For example, if the monitoring point is located near a starting electric motor, at this event you will have a high current draw, equal to the starting current required by the machine, which will result in a lowering of the voltage sensed by the PQ monitor (due to the increased voltage drop across the impedances of the system) and a raising of the absorbed reactive power. This will give you a positive deflection of the trend of $Q$.

In the case of upstream event, the load that caused the event will be in parallel to the load 'victim' which is located downstream of the monitoring point. The load 'victim' will maintain its phase angle, but the voltage on it will decrease, and, in a more or less relevant way, the current value will decrease too. Since the reactive power is proportional to these two quantities, the reactive power in transit absorbed by the load will decrease. For example, a ground fault in the neighborhood, upstream of a load, will result in a voltage drop which corresponds to a negative deflection measured.

In case of capacitive loads, for which therefore the reactive power is generated and its direction is opposite to that of reference for the active power, the situation is similar, but in case of event downstream is not sufficient, there is a positive deflection of reactive power, but this will also change its sign. Monitoring a capacitive system prevailing with respect to any resistive and/or inductive load, a disconnection of the capacitor bank $(Q<0)$, will cause a reduction of the power factor associated with the load, and then an instantaneous positive deflection of the reactive power which will now be equal to that absorbed $(Q>0)$ from the resistive-inductive load.

The method is summarized in the algorithm, presented in Fig. 8.
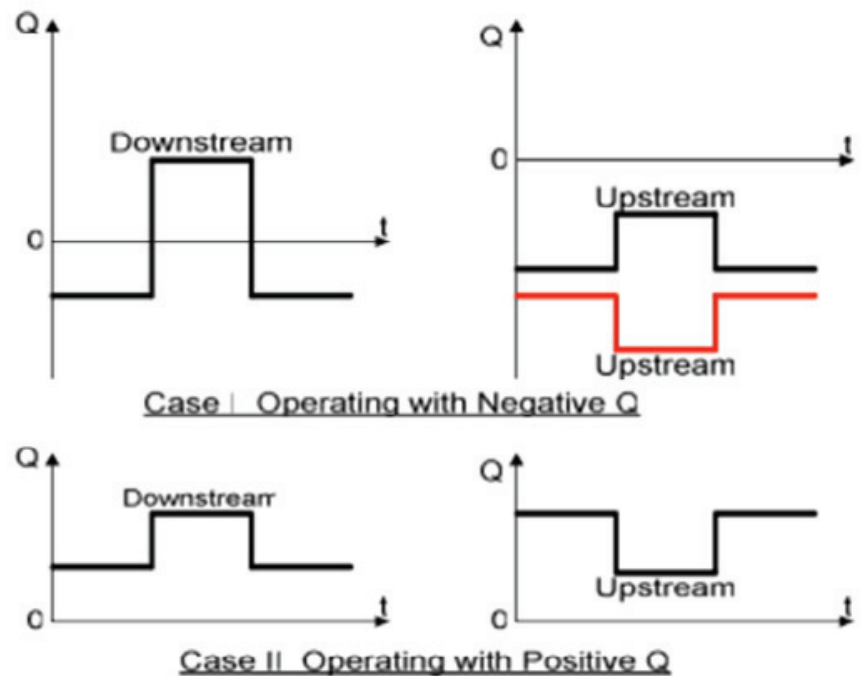

Fig. 8. Algorithm for the detection of sag 


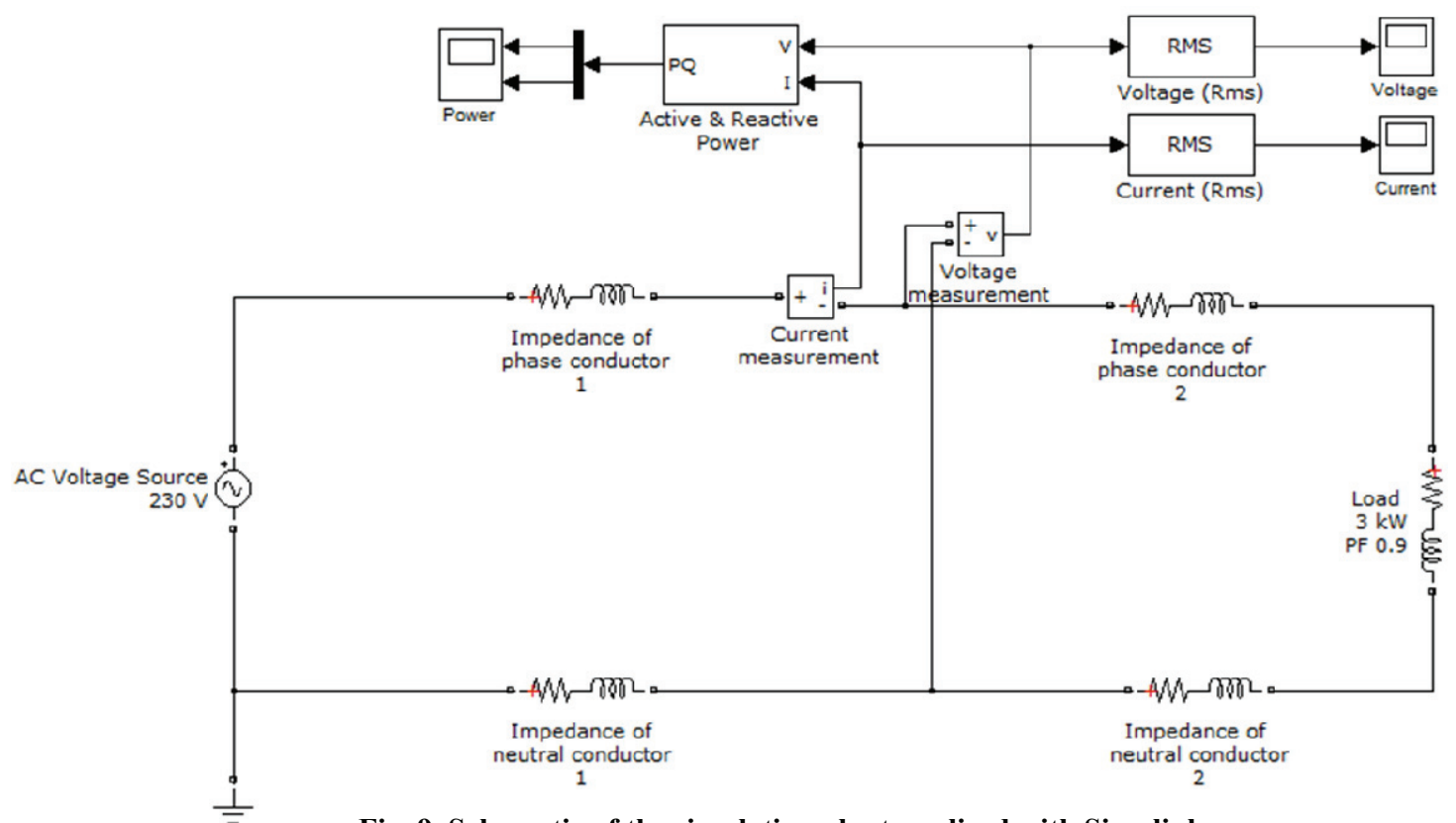

Fig. 9. Schematic of the simulation plant, realized with Simulink

For the algorithm validation a reference network has been used and the causes of voltage sags have been later implemented on it.

The reference network is a schematic of a singlephase low voltage network. Thus a single-phase $230 \mathrm{~V}$ power generator is represented.

It was decided to use a bipolar copper cable with section $2 \cdot 2,5 \mathrm{~mm}^{2}$. The length is assumed to be $50 \mathrm{~m}$ and the monitoring device (a voltmeter plus an ammeter) is inserted at a distance of $25 \mathrm{~m}$ from the transformer room.

In the calculation of the line impedances the mutual impedances between the conductors have been neglected.

The test plant is shown in Fig. 9.
The network feeds a $3 \mathrm{~kW}$ user with power factor equal to 0,9 (e.g. an apartment).

The simulations were divided into two parts: the first concerns the faults and the second the switching on and off of loads. All cases of disturbances have been implemented both upstream and downstream of the monitoring point.

As an example we report two cases: a short circuit and an insertion of an asynchronous motor.

The display interval of events is constant and equal to $1 \mathrm{~s}$. The insertion of the disturbance is set for all events at time $0,2 \mathrm{~s}$.

\section{Downstream fault}

The downstream fault was simulated according to the configuration presented in Fig. 10.

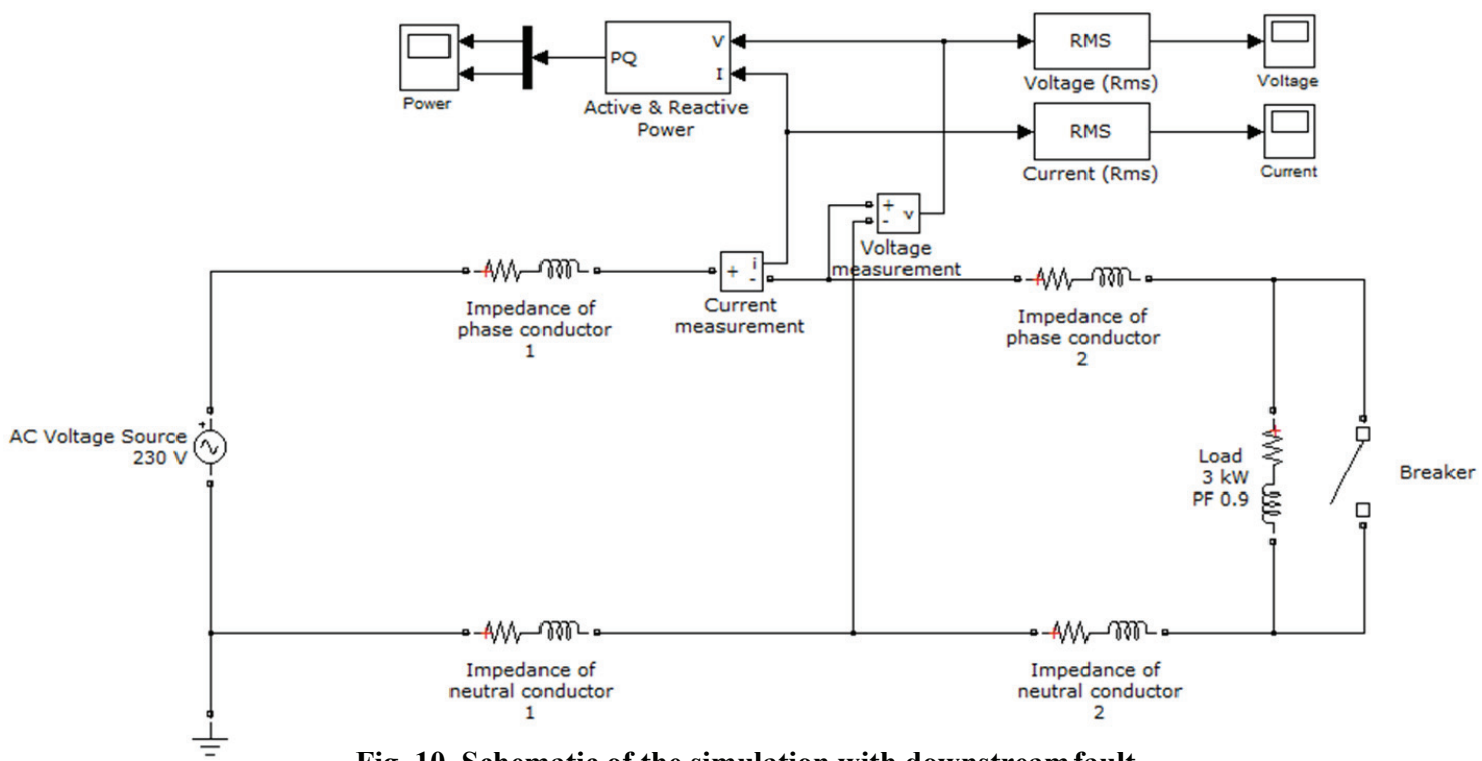

Fig. 10. Schematic of the simulation with downstream fault 


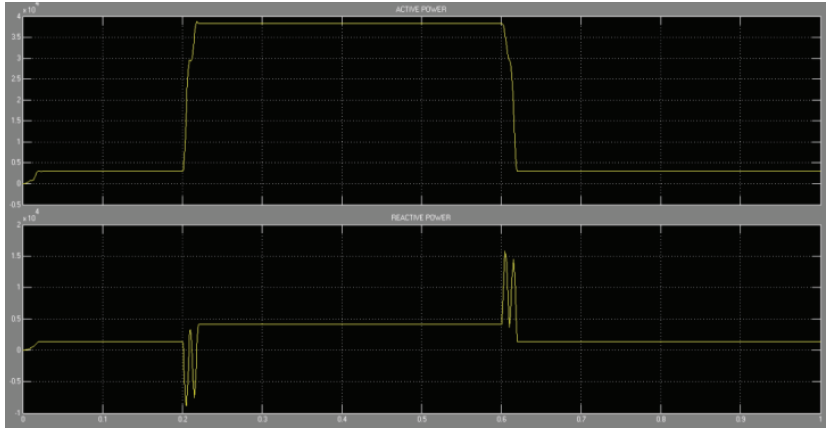

a)

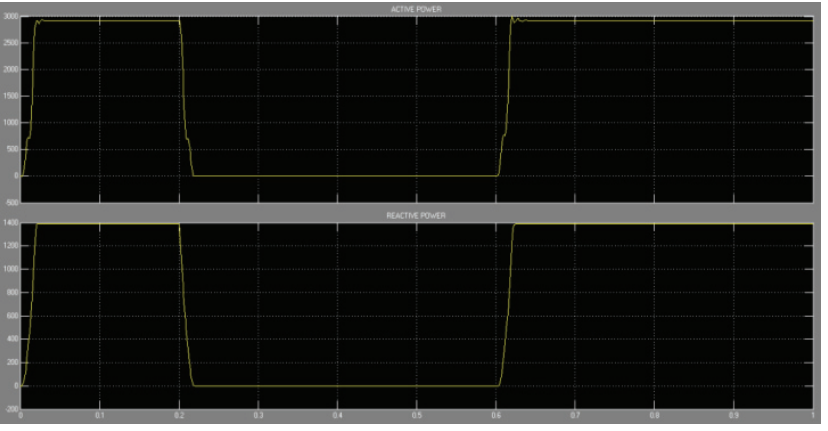

b)

Fig. 11. Simulation of the downstream (a) and upstream (b) fault (Active Power is expressed in W and Reactive Power in VAR)

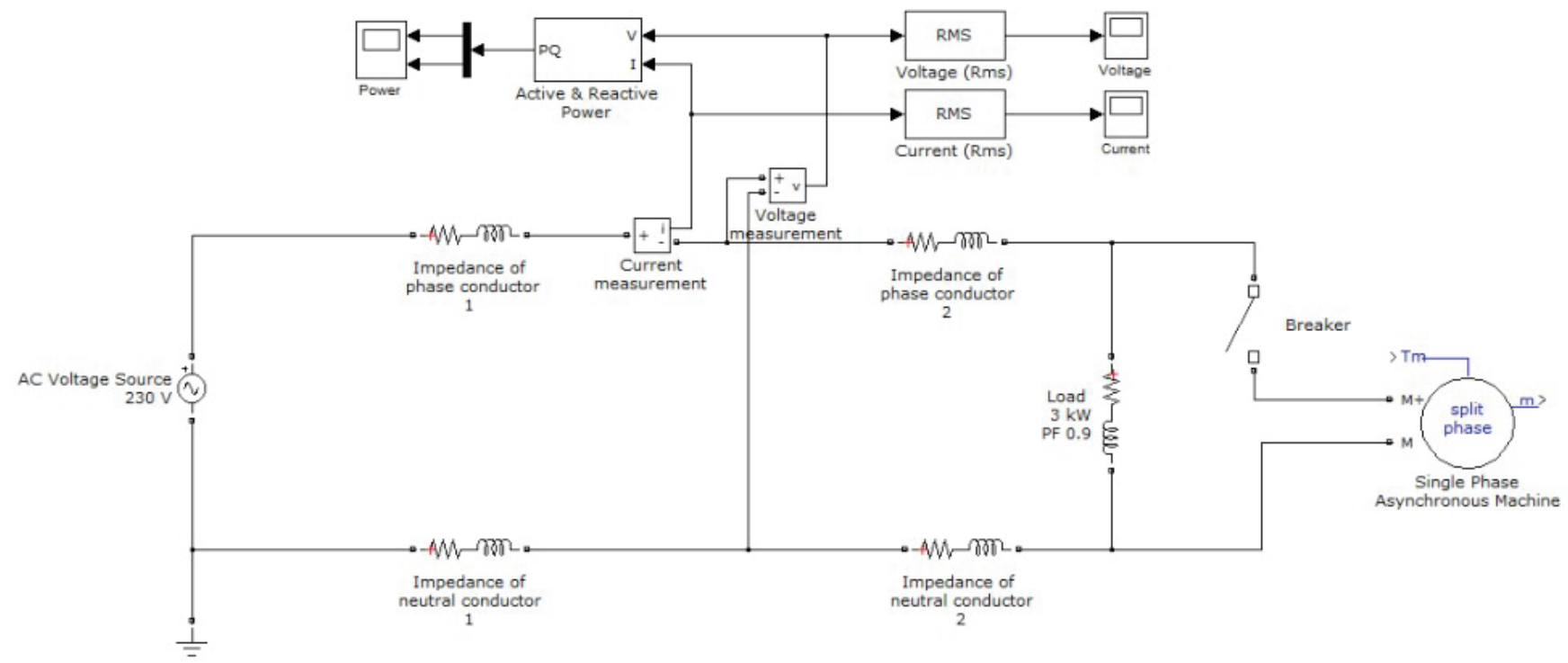

Fig. 12. Schematic with the insertion of a downstream motor

As you can see from the graphs (Fig. 11a), the method of reactive power is respected.

\section{Upstream failure}

Similarly, the failure was simulated upstream of the monitoring point. The graph (Fig. $11 \mathrm{~b}$ ) shows that the reactive power during the fault undergoes a sharp decrease. The method of the reactive power is therefore verified.

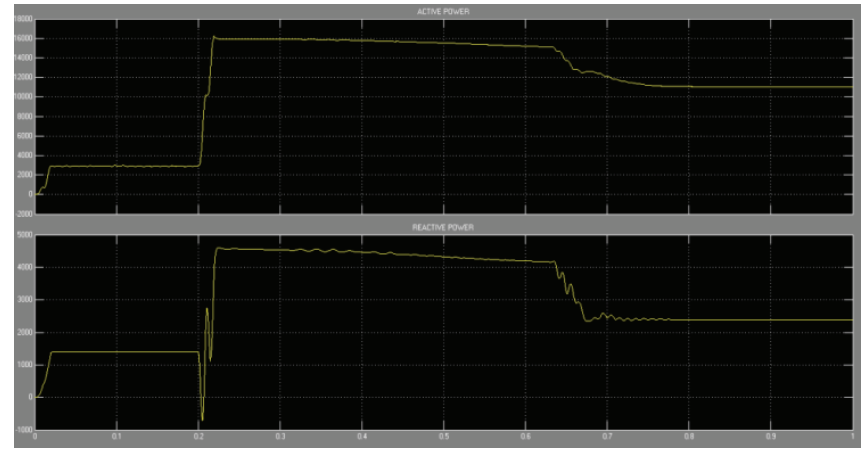

a)

\section{Insertion of a downstream motor}

Downstream of the monitoring point $3 \mathrm{kVA}$ asynchronous motor has been inserted, according to the configuration presented in Fig. 12.

As it can be seen from the graph in Fig. $13 a$, the reactive power undergoes a sharp increase during the starting transient. The method is therefore verified.

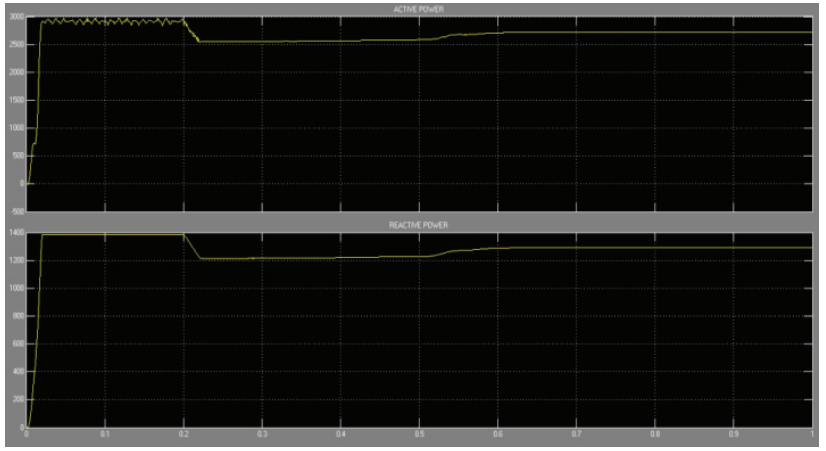

b)

Fig. 13. Simulation with the insertion of a motor downstream $(a)$ and upstream (b) (Active Power is expressed in $\mathrm{W}$ and Reactive Power in VAR) 


\section{Insertion of a motor upstream}

Similarly, it has been simulated the insertion of the same motor upstream of the PQ monitoring point.

The following graph shows the trends of the active and reactive power.

As it can be seen in Fig. $13 b$, even in the latter case the method is verified because there is a decrease in the reactive power during the starting transient.

\section{Multipoint Measurements}

We are now developing the second stage of the project consisting of designing and building the sensors network. In this case we have a number of SPQ devices interconnected with each other via two different types of connection: wireless ZigBee protocol for those topologies that require measurement points that are not too far away. In fact the protocol is designed for lengths from 10 to $75 \mathrm{~m}$ depending on the location of the devices, this choice may also be necessary when it is impossible to do wiring connections. A second possibility is to place the devices on a wired network, this allows you to overcome the problems related to the distances of the measuring points, but this second solution requires an existing network or the ability to create one with an increase in costs which is non-negligible.

The sensor network collects data and through special algorithms filters out the network anomalies. Data are then sent to a router where they are stored in a database and reported with appropriate warnings. The algorithm discussed above is implemented within the sensors network in order to obtain a distributed measurement system to detect the directionality of sags.

The algorithm for the detection of sags is implemented within individual measurement nodes, while that for the detection of directionality is performed in the router. The following are the basic steps of the algorithm.

1. Trigger Event: the quantity under control for the detection of the event is the amplitude of the line voltage. The condition of the trigger event is $V\left(t^{\prime}\right)<90 \% \mathrm{~V}$, with $\mathrm{t}^{\prime}$ moment in which the sag occurs $\rightarrow V\left(t^{\prime}\right)=$ Vsag and $V=$ Vpresag. $V$ was set to the value declared by the regulations.

2. Processing of Reactive Power: the $I(t)$ and $V(t)$ are acquired in real-time. Also $Q(t)$ is calculated. When the sag event occurs $Q\left(t^{\prime}\right)$ is calculated.
The device is equipped with a backup battery for operation during the voltage dip.

3. Detection: implementation of the algorithm illustrated in the previous section. Then signal $S$ occurs: 0 (upstream) or 1 (downstream) of the $i$-th measurement point.

The measuring points are numbered in a consecutive way, starting from 1 for measurement point close to the point of delivery.

4. Comparison with the 2 adjacent measurement points (implemented within the router): if at a certain node $S(i)=1$, a recursive comparison with the node $i+1$ will be done. The first node in which the condition $S(j)=0$ locates the cause of the gap between $j-1$ and $j$. Checking this condition for $j<i$ is not interesting, if we're looking for the cause of the event ' 1 ' (event downstream) because I need to move on nodes "far" from the delivery point. If $j=n$ and $S(j)=1$, the cause of the sag is among the loads that are located downstream from the last measurement point.

If at a node $S(i)=0$ the comparison will be done with the nodes $j<i$. The first node in which $S(j-1)=1$, means that the cause of the sag is between the nodes $j-1$ and $j$. At this point, checking this condition for $j>i$ is not interesting if we are looking for the cause of the event 0 (upstream event). Only nodes in the neighborhood need to be checked. If for $i=1 S(j)=0$ the cause of the sag is in the system upstream of my measurement area.

\section{Conclusion}

In this work a wireless sensors-based network is presented to perform data acquisition and signal processing of voltage and current in electrical systems, in order to evaluate the indices of PQ in accordance with IEC 61000-4-30 and IEC 50160. The wireless solution adopted, based on the Zigbee protocol, together with the choices made of the measuring devices and processing makes the measurement system achieve a good compromise between good performance and very low costs.

This is important for a large-scale use in all situations that require a detailed analysis of the electrical system from the point of view of the characterization of $\mathrm{PQ}$, without the adoption of expensive specialized tools. It is also a good starting point to face with different situations in the future. The tests carried out on the prototype node confirm the validity of the device also in terms of linearity, bandwidth and reliability. 
In the second phase of the project the wireless network will be completed in order to implement the algorithm for analysis of disturbances directionality. Also a data storage system will be implemented (not yet discussed here). Finally, we are working on a new version of the sensor's prototype that will significantly reduce its size, consumption and costs.

In this article, an overview of the main disturbances affecting the electrical power system operation has been presented. Harmonic issues have been investigated more deeply. Additionally, monitoring and evaluating the power quality from the point of view of harmonic disturbances have been introduced. The necessity to have a standard method to identify the sources of electrical power quality deterioration, and to evaluate accurately the actual proportion of responsibility of each of the players involved has been underlined.

\section{References}

1. CEI EN 61000-4-30, Compatibilità elettromagnetica (EMC). Parte 4- 30: Tecniche di prova e di misura - Metodi di misura della qualità della potenza.

2. IEEE Std. 1159, IEEE Recommended Practice for Monitoring Electric Power Quality,1995.
3. CEI EN 50160, Caratteristiche della tensione fornita dalle reti pubbliche di distribuzione dell'energia elettrica, 2011-05

4. Lysov, N.Yu. Razrabotka i issledovanie bystrodeistvuyushchikh intellektual'nykh privodov mekhatronnykh system [Development and research of highperformance intelligent drive mechatronic systems]. Mekhatronika, 2001, no. 2. pp. 35-43.

5. Clarence, W., Silva I. Mechatronic systems: devices, design, control, operation and monitoring. Mechatronics, 2008, 545 p.

6. Ferrari, P., Flammini A., Marioli D. and Taroni A. A low-cost Internet enabled smart sensor. Proc. of IEEE Sensors 2002, The First IEEE International Conference on Sensors, Orlando, Florida, USA, 12-14 June 2002, vol. 55, pp. 1324-1336.

7. Werter, M.J. A digital phase-locked loop for frequency detection. Circuits and Systems, 1995, Proceedings., Proceedings of the 38th Midwest Symposium on, Rio de Janeiro, Brazil, 13-16 Aug 1995, vol. 2, 1995, pp. 1252-1255.

8. Dzhekson R.G. Noveishie datchiki [The latest sensors]. Moskva: Tekhnosfera, 2007, 384 p.

9. Cataliotti, A., Cosentino V. and Nuccio S. A virtual instrument for the measurement of IEEE Std. 1459 - 2000 power quantities, Instrumentation and Measurement, IEEE Transactions, vol. 57, Jan. 2008, pp. 85-94. 